\title{
Contribución del epifitismo accidental a la distribución de especies de plantas vasculares en un bosque templado lluvioso
}

\section{Contribution of accidental epiphytism to the vascular plants distribution in a southern temperate rainforest}

\author{
Rosario Guzmán-Marín* \& Alfredo Saldaña \\ Departamento de Botánica, Facultad de Ciencias Naturales y Oceanográficas, Universidad de Concepción, Casilla 160-C, \\ Concepción, Chile. \\ *rguzmanma@udec.cl
}

\begin{abstract}
In a Valdivian forest stand, we identified vascular plant species occurring as accidental epiphytes, and we determined the contribution of accidental epiphytism on their local abundance. Four species were found as accidental epiphytes, being the fern Blechnum mochaenum the species showing the higher abundance proportion as accidental epiphyte (40 $\%)$. Unexpectedly, individuals of this species occurring as accidental epiphytes did not differ in functional traits related to nutrient availability (LMA-leaf mass ratio- and leaf $\mathrm{C} / \mathrm{N}$ ratio) from those that usually occur on the forest ground. As a shade tolerant and slow growing species, $B$. mochaenum apparently is not affected by the environmental restrictions of the epiphytic habitat.
\end{abstract}

Las epífitas vasculares constituyen un componente florístico importante en muchos ecosistemas forestales (Benzing 1990, Zotz \& Andrade 2002, Zotz 2005, Taylor \& Burns 2015). Benzing (1990) las clasifica dentro de tres categorías funcionales: obligadas, facultativas y accidentales. Las primeras son las que presentan todo su ciclo de vida en el hábito epífito. Las "facultativas" pueden presentarse como epífitas o habitando el suelo del bosque, mientras que las "accidentales" corresponden a especies principalmente enraizadas al suelo y que no poseen modificaciones en sus estructuras o en su fisiología que reflejen estar adaptadas para la vida en el dosel (Benzing 1990, Burns 2010). El epifitismo accidental puede otorgar a las plantas la posibilidad de establecerse fuera de la competencia con otras en el suelo, ampliando así su nicho y distribución a escala local. En general, se desconoce la contribución de dicha estrategia a la distribución y abundancia de las especies del sotobosque en bosques templados del sur de Sudamérica. Las plantas epífitas accidentales también podrían presentar diferencias en la expresión de algunos rasgos funcionales en relación con las que están establecidas en el suelo, puesto que están sometidas a condiciones microambientales propias del hábitat epífito. Por ejemplo, se esperaría que en un ambiente pobre en nutrientes como el sustrato epífito (Benzing 1990, Zotz \& Andrade 2002), la relación C/N foliar y masa foliar específica (LMA) sean más altas que en uno con mayor disponibilidad, puesto que en un ambiente pobre en nutrientes la estrategia de desarrollo tiende a ser el crecimiento lento, optimizando la formación de hojas longevas (mayor resistencia mecánica, mayor cutícula, menor palatabilidad, etc.) (Lambers et al. 2008).

Rodales maduros que representen bien la expresión máxima de diversidad vegetal de una comunidad de bosque templado lluvioso presentan una estructura que ofrece gran variedad de micrositios disponibles para evaluar el establecimiento de las epífitas accidentales. En este tipo forestal, donde la riqueza de plantas vasculares epífitas es relativamente alta para un bosque templado (Muñoz-Schick 1980, Saldaña et al. 2014), comparado con bosques templados del hemisferio norte (Zotz 2005), se ha observado que algunas especies del sotobosque se presentan como epífitas accidentales. Como no se ha caracterizado cuantitativamente este patrón ecológico en este tipo de bosque en particular, y hasta donde sabemos, en ningún otro ecosistema forestal en Chile, evaluamos el epifitismo accidental en un bosque templado lluvioso (Parque Nacional Puyehue, $40^{\circ} 30^{\prime} \mathrm{S}, 71^{\circ} 50^{\prime} \mathrm{O}$ ), con el fin de: i) identificar las especies de plantas vasculares que presentan epifitismo accidental, ii) evaluar la contribución del hábitat epífito accidental en la abundancia de estas especies en el bosque, y iii) evaluar si los individuos de estas especies que utilizan el hábitat epífito difieren morfológica y funcionalmente de los que se establecen en el suelo. Hipotetizamos que para las especies que presentan epifitismo accidental, los individuos epífitos difieren significativamente de los establecidos en el suelo en los atributos funcionales razón $\mathrm{C} / \mathrm{N}$ foliar y LMA, 
respondiendo a la menor disponibilidad de nutrientes que debiera presentar el sustrato epífito. Para esto último también evaluamos la razón $\mathrm{C} / \mathrm{N}$ del sustrato epífito y del suelo.

La zona estudiada se encuentra a una altura de 350$400 \mathrm{msnm}$, y presenta un promedio de precipitación anual de $2.800 \mathrm{~mm}$ y $9,8{ }^{\circ} \mathrm{C}$ de temperatura (Dorsch 2003). La vegetación corresponde principalmente a bosque laurifolio templado interior, con un dosel dominado por Nothofagus dombeyi, Eucryphia cordifolia, Weinmannia trichosperma, Laureliopsis philippiana y Dasyphyllum diacanthoides (Luebert \& Pliscoff 2006), el sotobosque dominado por especies arbustivas como Fuchsia magellanica, Chusquea quila, Myrceugenia planipes, Azara lanceolata y Mitraria coccinea (Muñoz-Schick 1980), y entre las herbáceas las más abundantes son los helechos Blechnum hastatum, B. chilense y B. mochaenum (Saldaña et al. 2005). En 40 parcelas de $25 \mathrm{~m}^{2}$ ubicadas al azar al interior del bosque se determinó la frecuencia de especies herbáceas, arbóreas y arbustivas. Todas las parcelas estaban en un rango de entre $20-25 \%$ de apertura del dosel, tanto en el sotobosque como en el hábitat epifito. En estas parcelas fueron también identificadas las epífitas accidentales y se estimó su frecuencia como epífita y en el suelo. La identificación se realizó en base a la descripción de la flora de la zona (i.e. Muñoz-Schick 1980; Marticorena et al. 2010). De la especie que presentó mayor abundancia como epífita accidental (B. mochaenum) se colectaron 40 individuos al azar (20 epífitos y $20 \mathrm{del}$ suelo) para comparar los rasgos funcionales LMA y razón $\mathrm{C} / \mathrm{N}$ foliar. En el laboratorio las hojas fueron secadas $48 \mathrm{~h}$ a $70{ }^{\circ} \mathrm{C}$ y pesadas en una balanza analítica, para estimar LMA (peso seco/área foliar). Previamente, se determinó el área foliar con el programa Image J (http://imagej.nih.gov/ij). La razón $\mathrm{C} / \mathrm{N}$ foliar y del sustrato de una submuestra $(\mathrm{n}=15$ réplicas $\mathrm{x}$ hábitat para hojas; $\mathrm{n}=5 \mathrm{x}$ hábitat para sustrato), se analizaron en el Laboratorio de Suelos de la Facultad de Agronomía de la Universidad de Concepción. La proporción de la abundancia que corresponde al hábitat epífito accidental se expresó en base a la frecuencia por especie $\mathrm{y}$ hábitat. Los valores promedio de LMA entre individuos epífitos accidentales y del suelo (control) de B. mochaenum se compararon con t-Student, y para la razón $\mathrm{C} / \mathrm{N}$ foliar $\mathrm{y}$ de sustrato con el test U Mann-Whitney. Los análisis fueron realizados con el software JMP 10 (SAS-Institute, EEUU).

De las veinticinco especies encontradas en el muestreo, cuatro especies nativas presentaron el hábitat de epífita accidental: Blechnum mochaenum G. Kunkel, Chusquea quila Kunth, Megalastrum spectabile (Kaulf.) A.R. Sm. \& R.C. Moran y Myrceugenia planipes (Hook. \& Arn.) O. Berg. En particular B. mochaenum fue la más frecuente como epífita accidental (45\% de su abundancia total; Fig. 1). Las tres especies restantes presentan el hábito epífito con menor frecuencia ( $C$. quila $10 \%$, M. planipes $2,5 \%$ y M. spectabile $2,5 \%$ ). En el caso de B. mochaenum, ninguno de los rasgos funcionales evaluados mostró diferencias significativas entre individuos que crecen en el suelo o como epífito
(Tabla 1). El suelo epífito y del bosque tampoco mostraron diferencias significativas en razón $\mathrm{C} / \mathrm{N}(p=0,401)$.

En el sitio de estudio el número de especies que presentan epifitismo accidental no es muy alto, pero es relativamente comparable con el número de especies encontrado en algunos bosques tropicales (Nieder et al. 2000) o en bosques templados del hemisferio norte (Burns 2008). En el presente estudio las epífitas accidentales son especies que habitan el sotobosque, cuyos hábitos de crecimiento son herbáceo, arbustivo, y arbustivo/trepador. La especie más recurrente como epífita accidental (B. mochaenum) es un helecho herbáceo sombra-tolerante (Saldaña et al. 2005), que al parecer estaría colonizando el sustrato epífito desde el suelo adyacente alárbol. Probablemente este hecho se ve favorecido por la gran producción y capacidad de dispersión de esporas que presentan en general los helechos (Tryon 1970). Las esporas son liberadas al aire donde pueden permanecer por largo tiempo, dependiendo de las condiciones (temperatura, viento, humedad, precipitaciones) (Noguez 2012). Dado este modo de dispersión, la probabilidad de que esporas de los individuos de B. mochaenum cercanos a los micrositios del hábitat epífito logren colonizar y establecerse con éxito es bastante alta. Como consecuencia, para esta especie el hábitat epífito contribuye de manera importante a su distribución y abundancia en un bosque templado lluvioso maduro. Contrario a lo esperado, los individuos epífitos accidentales de $B$. mochaenum no presentaron diferencias en rasgos funcionales con los establecidos en el suelo. El que no haya diferencias entre los dos tipos de hábitat en la disponibilidad de algunos recursos como la luz o los nutrientes, refleja que los patrones de respuesta funcional en ambos ambientes sean similares. Esto no es extraño dada la estrategia de crecimiento lento que posee la especie (Saldaña et al. 2005). Además, los micrositios en donde se albergan los individuos epífitos accidentales se crean a partir de la

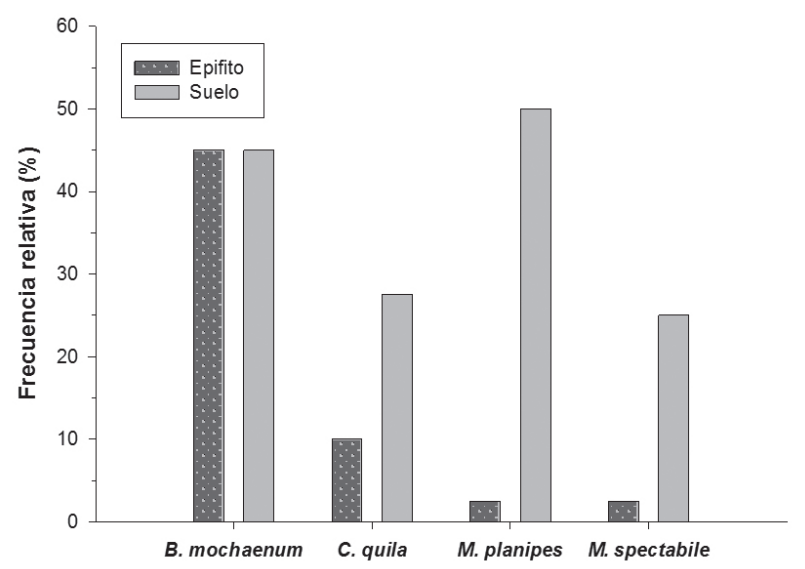

FIgURA 1. Abundancia (frecuencia relativa) de las especies presentes como epífitas accidentales, comparada con su abundancia en el suelo, en un bosque templado lluvioso del sur de Chile. I Abundance (relative frequency) of species that occur as accidental epiphytes, compared with their abundance on the ground, in a temperate rainforest in southern Chile. 
descomposición de los mismos componentes que están en el suelo, ya que se encuentran bajo el mismo dosel.

A priori se esperaba encontrar mayor incidencia de epifitismo accidental por parte de la vegetación en el sitio de estudio, por ejemplo de especies herbáceas, ya sea nativas o introducidas. Sin embargo, observamos que el epifitismo accidental en este rodal de bosque maduro no es muy común. La estructura de la vegetación, al ser un bosque maduro, dejaría poco espacio para la llegada de epífitas accidentales, principalmente por la baja probabilidad de dispersión de propágulos, e.g. de especies herbáceas presentes en claros grandes, o porque las condiciones de germinación y establecimiento no son las adecuadas (e.g. baja apertura de dosel). Probablemente los ensambles vegetales que han sufrido perturbaciones, ya sean autogénicas (caída de árboles o ramas) o de forma externa (tala), podrían quedar más expuestos a la colonización de plantas exóticas o al auge de las pertenecientes al sotobosque por sobre las dominantes del dosel, lo cual podría cambiar el patrón reportado en este estudio. Hasta la fecha, no se han estudiado este tipo de formaciones vegetacionales y cómo podría estar siendo la colonización del hábitat epífito en estos escenarios ecológicos, por lo que podríamos encontrar plantas en comportamiento de epífitas accidentales favorecidas por la perturbación. En este sentido, nuestro estudio abre las puertas para investigar este tipo de patrón en otras formaciones vegetacionales del cono sur y ver qué sucede ante otras circunstancias como las anteriormente descritas.

TABLA 1. Valores promedio ( \pm ES) de LMA y razón C/N foliar de individuos de B. mochaenum establecidos como epífitos y en el suelo del bosque, y razón $\mathrm{C} / \mathrm{N}$ del sustrato de cada tipo de micrositio. N.S.: diferencias no significativas ( ${ }^{\dagger} \mathrm{t}$ test; *U Mann-Whitney). / Mean values ( \pm $\mathrm{SE}$ ) of LMA and leaf $\mathrm{C} / \mathrm{N}$ ratio of $B$. mochaenum individuals occurring in epiphytic and ground forest microsites, and substrate $\mathrm{C} / \mathrm{N}$ ratio in each microsite. N.S.: not significant (†t test; ${ }^{\mathrm{U}} \mathrm{U}-\mathrm{Mann}-$ Whitney U test).

\begin{tabular}{|c|c|c|}
\hline & EpíFITO & SuELo \\
\hline $\operatorname{LMA}\left(\mathrm{g} \mathrm{cm}^{-2}\right)^{\text {N.S. } \dagger}$ & $6,27 \mathrm{E}^{-3} \pm 0,24 \mathrm{E}^{-3}$ & $6,32 \mathrm{E}^{-3} \pm 0,24 \mathrm{E}^{-3}$ \\
\hline $\mathrm{C} / \mathrm{N}$ foliar ${ }^{\mathrm{N} . S . \dagger}$ & $24,27 \pm 0,58$ & $24,88 \pm 0,58$ \\
\hline $\mathrm{C} / \mathrm{N}$ sustrato ${ }^{\mathrm{N} . \mathrm{S} .}$. & $13,31 \pm 0,75$ & $12,37 \pm 0,75$ \\
\hline
\end{tabular}

\section{AGRADECIMIENTOS}

A N. Viveros, D. Rodríguez, F. Carrasco, L. Vecchi, M. Moreno. Proyecto Fondecyt 1140455.

\section{REFERENCIAS}

Benzing, D.H. 1990. Vascular epiphytes. General biology and related biota. Cambridge University Press. 354 pp.

Burns, K.C. 2008. Meta-community structure of vascular epiphytes in a temperate rainforest. Botany 86(11): 1252-1259.

BuRNS, K.C. 2010. How arboreal are epiphytes? A null model for Benzing's classifications. New Zealand Journal of Botany 48 (3-4): 185-191.

Dorsch, K. 2003. Hydrogeologische Untersuchungen der Geothermalfelder von Puyehue und Cordón Caulle, Chile. Dissertation, Ludwig-Maximilians-Universität, München, Germany.

Lambers, H., Raven, J.A., Shaver, G.R., Smith, S.E. 2008. Plant nutrient-acquisition strategies change with soil age. Trends in Ecology \& Evolution 23(2): 95-103.

Luebert, F., Pliscoff, P. 2006. Sinopsis bioclimática y vegetacional de Chile. Editorial Universitaria, Santiago. 316 pp.

Marticorena, A., Alarcón, D., Abello, L., Atala, C. 2010. Guía de Campo, Plantas trepadoras, epífitas y parásitas nativas de Chile. Ediciones Corporación Chilena de la Madera, Concepción. 290 pp.

MuÑoz-Schick, M. 1980. Flora del parque nacional Puyehue.
Editorial Universitaria, Santiago. 557 pp.

Nieder, J., Engwald, S., Klawun, M., Barthlott, W. 2000. Spatial distribution of vascular epiphytes (including Hemiepiphytes) in a Lowland Amazonian Rain Forest (Surumoni Crane Plot) of Southern Venezuela 1. Biotropica 32(3): 385-396.

Noguez, B.F.G. 2012. Composición florística y variación estacional de la lluvia de esporas de helechos en el Bosque Mesófilo de Río Malila, Municipio de Molango, Hidalgo. Tesis. Magíster en Biología. Universidad Autónoma Metropolitana Iztapalapa, Ciudad de México, México. 150 pp.

Saldaña, A., Gianoli, E., Lusk, C.H. 2005. Ecophysiological responses to light availability in three Blechnum species (Pteridophyta, Blechnaceae) of different ecological breadth. Oecologia 145(2): 251-256.

Saldaña, A., Parra, M.J., Flores-Bavestrello, A., Corcuera, L.J., Bravo, L.A. 2014. Effects of forest successional status on microenvironmental conditions, diversity, and distribution of filmy fern species in a temperate rainforest. Plant SpeciesBiology 29(3): 253-262.

TAYlor, A., Burns, K. 2015. Epiphyte community development throughout tree ontogeny: an island ontogeny framework. Journal of Vegetation Science 26 (5): 902-910.

TRYON, R. 1970. Development and evolution of fern floras of oceanic islands. Biotropica 2(2): 76-84.

Zotz, G. 2005. Vascular epiphytes in the temperate zones - a review. Plant Ecology 176: 173-183.

Zotz, G., Andrade, J.L. 2002. La ecología y la fisiología de las epífitas y hemiepífitas. En: Guariguata, M.R., Kattan, G.H. (ed.). Ecología y conservación de bosques neotropicales. Ediciones LUR, Cartago. pp. 271-296.

Recibido: 25.11.2016

Aceptado: 21.04.2017 